Case Report

\title{
Acute Immune-Mediated Thrombocytopenia due to Oxaliplatin and Irinotecan Therapy
}

\author{
Eric L. Tam (D, Padma L. Draksharam, Jennifer A. Park, and Gurinder S. Sidhu \\ Department of Medicine, Division of Hematology and Oncology, State University of New York Downstate Medical Center, \\ Brooklyn, USA \\ Correspondence should be addressed to Gurinder S. Sidhu; gurisidhu@gmail.com
}

Received 7 June 2019; Revised 26 September 2019; Accepted 12 October 2019; Published 4 November 2019

Academic Editor: Katsuhiro Tanaka

Copyright (c) 2019 Eric L. Tam et al. This is an open access article distributed under the Creative Commons Attribution License, which permits unrestricted use, distribution, and reproduction in any medium, provided the original work is properly cited.

\begin{abstract}
We describe a case of a 63-year-old woman with advanced colon cancer and liver metastases who was treated with fluorouracil, leucovorin, and oxaliplatin (FOLFOX) and cetuximab chemotherapy. She tolerated 13 cycles of chemotherapy without any significant hematological side effects, but after the 14th cycle, she developed melena and was admitted for severe thrombocytopenia. After supportive care, the platelet counts rapidly improved to $76,000 / \mu \mathrm{L}$. Upon initiation of FOLFIRI and cetuximab chemotherapy, she again developed rectal bleeding and severe thrombocytopenia with a platelet count of $6000 / \mu \mathrm{L}$. Lab testing was positive for oxaliplatin and irinotecan drug-dependent platelet antibodies on flow cytometry assay. Druginduced thrombocytopenia (DITP) is associated with several classes of drugs with several proposed underlying mechanisms. Prospective studies are needed to further address different mechanisms of drug-induced thrombocytopenia.
\end{abstract}

\section{Introduction}

Drug-induced thrombocytopenia (DITP) occurs frequently but can be misdiagnosed or overlooked. In patients with cancer, many chemotherapeutic agents can cause thrombocytopenia due to bone marrow suppression; other causes of thrombocytopenia can include infection, autoimmune disease, and pseudothrombocytopenia $[1,2]$. Reported chemotherapeutic agents known to trigger immune drug-related thrombocytopenia are fludarabine, dactinomycin, cisplatin, oxaliplatin, and irinotecan $[3,4]$.

Fluorouracil, oxaliplatin, and irinotecan have been standard cytotoxic chemotherapeutic agents in advanced colorectal cancer. Oxaliplatin is a platinum derivative that has been widely used in patients with gastrointestinal malignancies including colorectal cancers. The combination of 5-fluorouracil, leucovorin, and oxaliplatin (FOLFOX) has been shown to increase survival rates and reduce the risk of disease progression in stage III colon cancer [5]. Following FOLFOX, thrombocytopenia was noted in $70 \%$ of patients, which is usually self-limited and assumed to be related to myelosuppression from oxaliplatin $[6,7]$. Irinotecan is a synthetic analog of camptothecin and often given in combination with 5- fluorouracil and leucovorin (FOLFIRI) and acts through inhibition of DNA topoisomerase I. Neutropenia and thrombocytopenia result from myelosuppression of the bone marrow and are usually mild $[3,8]$.

In this report, we describe a patient who developed acute immune-mediated thrombocytopenia to both oxaliplatin and irinotecan with the presence of bleeding symptoms during the treatment of metastatic colon cancer. Both chemotherapy agents were found to have positive platelet drugdependent antibodies (DDAbs).

\section{Case Presentation}

The patient is a 63-year-old female with metastatic colon cancer (KRAS wild type) to the liver and ascites, initially treated with palliative chemotherapy with FOLFOX and Cetuximab. Her platelet count prior to initiation of therapy was $102,000 / \mu \mathrm{L}$, hemoglobin was $9.7 \mathrm{~g} / \mathrm{dL}$, and total white blood cell count was $9,000 / \mu \mathrm{L}$. An infusion of FOLFOX was administered up to the 14th cycle of treatment with minimal effects on the platelet count. The patient was tolerating treatment well with stable disease on imaging. However, two days after the 14th treatment, she developed melena. Laboratory 
160

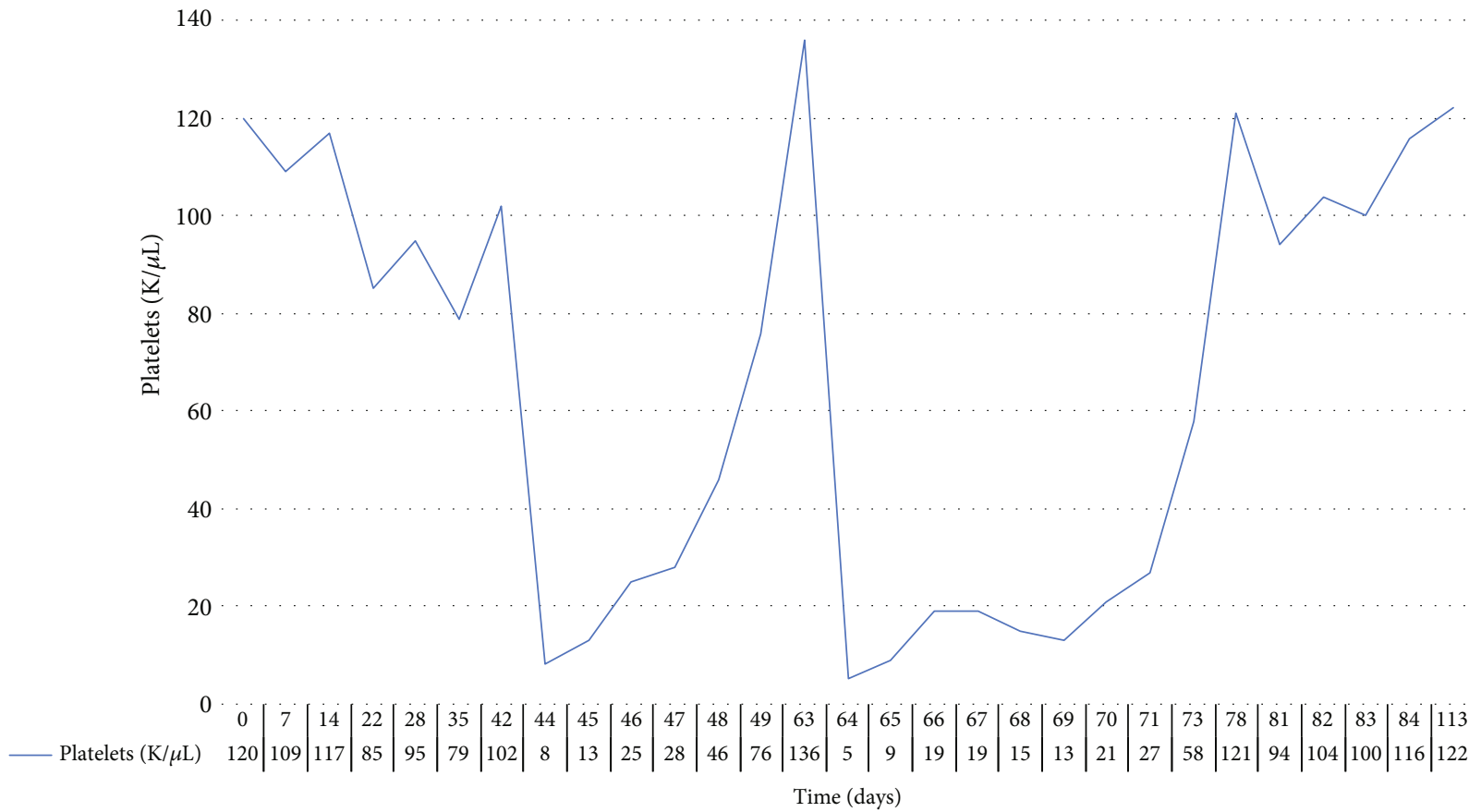

Figure 1: Platelet count over time for the two episodes of thrombocytopenia from oxaliplatin and irinotecan infusion with subsequent recovery.

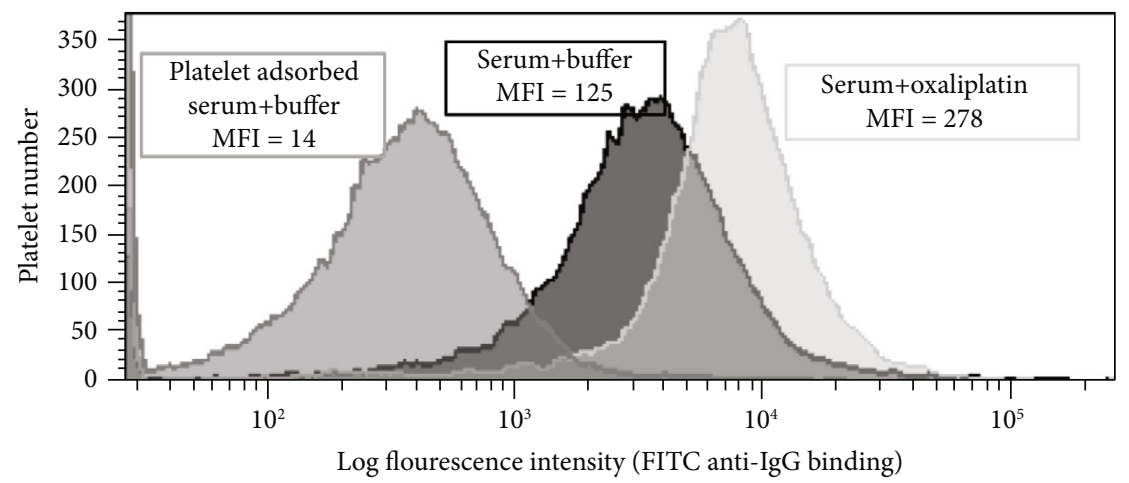

FIgURE 2: Detection of oxaliplatin-dependent platelet antibodies by flow cytometry. Platelets show high IgG binding when incubated with the patient's serum in the presence of oxaliplatin $(0.1 \mathrm{mg} / \mathrm{mL})$ (light gray histogram on the right) compared to platelets incubated with buffer/no drug present (dark histogram in the center). The numbers above each histogram are the median fluorescence intensity (MFI) values.

studies obtained showed platelets of $8,000 / \mu \mathrm{L}$, hemoglobin of $8.4 \mathrm{~g} / \mathrm{dL}$, and white blood cell count of $15,790 / \mu \mathrm{L}$. She was admitted to the hospital and transfused with two units of packed red blood cells and three units of platelets with minimal response to her laboratory values. A computed tomography (CT) scan of the abdomen and pelvis showed colitis, which was treated with intravenous ciprofloxacin and metronidazole. Five days following admission, platelet levels steadily improved reaching a level of $76,000 / \mu \mathrm{L}$. One month later, she was started on second-line chemotherapy, FOLFIRI, and cetuximab. Immediately following the first cycle of irinotecan, the patient again developed rectal bleeding, and on repeat, blood work was found to have a severe drop in platelets from $136,000 / \mu \mathrm{L}$ to $6,000 / \mu \mathrm{L}$ within 24 hours following the completion of the FOLFIRI infusion (Figure 1).

A peripheral blood sample was drawn immediately after the development of thrombocytopenia from FOLFIRI and sent for testing of platelet DDAbs. Drug-dependent platelet antibodies to oxaliplatin and irinotecan were detected in the patient's serum using flow cytometry techniques as previously described in the literature [6]. Interestingly, the patient's serum also showed positive reactions detected by flow cytometry in the absence of any drug but was also potentiated in the presence of oxaliplatin (Figure 2) and irinotecan. These results indicate the presence of oxaliplatin-dependent, irinotecan-dependent, and nondrug-dependent platelet- 
TABLE 1: Known cases of oxaliplatin and irinotecan drug-induced immune-mediated thrombocytopenia. IVIG=intravenous immunoglobulin.

\begin{tabular}{|c|c|c|c|c|c|c|}
\hline \multirow[t]{2}{*}{ Study } & \multirow[t]{2}{*}{ Drug } & \multirow{2}{*}{$\begin{array}{l}\text { Cycles prior } \\
\text { to DITP }\end{array}$} & \multicolumn{2}{|c|}{$\begin{array}{l}\text { Platelet count } \\
\quad(\text { per } \mu \mathrm{L})\end{array}$} & \multirow[t]{2}{*}{$\begin{array}{l}\text { Time to platelet } \\
\text { recovery (days) }\end{array}$} & \multirow[t]{2}{*}{ Treatment } \\
\hline & & & Initial & & & \\
\hline Akdeniz et al. [17] & Oxaliplatin & 12 & 151,000 & 20,000 & 6 & $\begin{array}{c}\text { Corticosteroids, platelets, } \\
\text { IVIG }\end{array}$ \\
\hline \multirow[t]{2}{*}{ Bautista et al. [6] } & Oxaliplatin & 3 & 226,000 & 4,000 & 12 & $\begin{array}{l}\text { Antihistamine, } \\
\text { corticosteroids, platelets }\end{array}$ \\
\hline & Oxaliplatin & 3 & 87,000 & 66,000 & 14 & Corticosteroids \\
\hline Buti et al. [18] & Oxaliplatin & 11 & 117,000 & 17,000 & NA & NA \\
\hline Cobo et al. [19] & Oxaliplatin & 12 & 202,000 & 7,000 & NA & NA \\
\hline \multirow{2}{*}{ Curtis et al. [8] } & Oxaliplatin & 17 & 150,000 & 6,000 & 7 & Platelets \\
\hline & Oxaliplatin & 10 & 136,000 & 6,000 & 21 & Observation \\
\hline & Oxaliplatin, irinotecan & 20 & 130,000 & 0 & NA & Corticosteroids \\
\hline Curtis et al. [10] & $\begin{array}{l}\text { Oxaliplatin, irinotecan, } \\
\text { leucovorin, dexamethasone, } \\
\text { diphenhydramine, } \\
\text { palonosetron }\end{array}$ & 18 & 157,000 & 5,000 & NA & Platelets \\
\hline Earle et al. [20] & Oxaliplatin & 1 & 99,000 & 6,000 & 14 & Platelets, corticosteroids \\
\hline Fontão-Wendel et al. [9] & Oxaliplatin & 7 & 164,000 & 5,000 & 3 & Corticosteroids, IVIG \\
\hline James et al. [14] & Oxaliplatin & 28 & 145,000 & 0 & 5 & Corticosteroids, platelets \\
\hline Koutras et al. [21] & Oxaliplatin & 14 & NA & NA & 2 & $\begin{array}{l}\text { Corticosteroids, } \\
\text { antihistamines, platelets }\end{array}$ \\
\hline Mirtsching et al. [11] & Irinotecan & NA & 347,000 & $\begin{array}{c}84- \\
100,000\end{array}$ & NA & Observation \\
\hline Mittal et al. [12] & Oxaliplatin & 8 & 175,000 & 4,000 & 9 & Platelets, corticosteroids \\
\hline Ohta et al. [22] & Oxaliplatin & 12 & 136,000 & 35,000 & 7 & Corticosteroids \\
\hline Pavic et al. [23] & Oxaliplatin & 1 & 253,000 & 5,000 & NA & Corticosteroids \\
\hline Saif et al. [24] & Oxaliplatin & 11 & 89,000 & 17,000 & 2 & Platelets \\
\hline Santodirocco et al. [25] & Oxaliplatin & 15 & 124,000 & 21,000 & 5 & Corticosteroids, platelets \\
\hline Schade et al. [26] & Oxaliplatin & 14 & 89,000 & 5,000 & 45 & Platelets \\
\hline Shao et al. [27] & Oxaliplatin & 24 & 163,000 & 4,000 & NA & Platelets \\
\hline Suh et al. [15] & Oxaliplatin & 11 & 113,000 & 3,000 & 60 & $\begin{array}{l}\text { Platelets, IVIG, } \\
\text { corticosteroids }\end{array}$ \\
\hline Suzuki et al. [28] & Oxaliplatin & 23 & 253,000 & 3,000 & 5 & Platelets, corticosteroids \\
\hline Taleghani et al. [29] & Oxaliplatin & 15 & 221,000 & 5,000 & 5 & Platelets, corticosteroids \\
\hline Tam et al. [this report] & Oxaliplatin, irinotecan & 14 & 102,000 & 8,000 & 5 & Corticosteroids, platelets \\
\hline Curtis et al. [30] & Leucovorin, palonosetron & 19 & 144 & 17 & 4 & Dexamethasone \\
\hline Pan et al. [31] & Oxaliplatin & 28 & NA & $<5,000$ & 4 & IVIG, dexamethasone \\
\hline
\end{tabular}

reactive antibodies. The patient's serum was also tested for fluorouracil and cetuximab-dependent platelet antibodies which were negative for both IgG and IgM DDAbs.

She was transfused with platelets and started on dexamethasone. She showed improvement, and platelet counts improved her baseline over the next few weeks. While treatment was on hold, her clinical condition deteriorated and she was not eligible for further lines of therapy. She chose to pursue the best supportive care and passed away in hospice.

\section{Discussion}

We present a case of isolated thrombocytopenia due oxaliplatin and irinotecan. Common mechanisms of thrombocytope- nia in this setting include direct toxicity of the bone marrow and immune-mediated thrombocytopenia caused by drugdependent antibodies [9]. In this case, thrombocytopenia was likely immune-mediated secondary to DDAbs. This is supported by the presentation of thrombocytopenia immediately after drug administration without evidence of any other cytopenias and laboratory conformation of oxaliplatin and irinotecan DDAbs. Table 1 shows some of the previously reported cases in the literature of oxaliplatin and/or irinotecan-induced immune-mediated thrombocytopenia, in patients with colorectal cancer. Curtis et al. recently reported thrombocytopenia [10] induced by both oxaliplatin and irinotecan. In the first case, the patient was treated with both oxaliplatin and irinotecan for 18 cycles prior to the 
development of DITP. In the second case, the patient developed hemolytic anemia concurrently with DITP. Our case remains unique because DITP resulted not only from exposure to oxaliplatin with FOLFOX after 14 cycles but also upon the very first exposure to irinotecan with FOLFIRI.

In our patient, platelet DDAbs were detected in the presence of both oxaliplatin and irinotecan. This suggests that the DDAbs to irinotecan were already present prior to irinotecan exposure and the same DDAb may be implicated, or oxaliplatin generated multiple DDAbs, with one specific for irinotecan. DDAbs can induce the destruction of platelets through immune complex production, autoantibody production, or hapten-dependent antibody response [11, 12]. Metabolites of oxaliplatin are also known to be reactive and may form covalent bonds to blood proteins and macromolecules creating neoepitopes which may have specific targets such as glycoprotein IIb/IIIa platelet membrane complex, complement proteins, and ADAMTS13 [13]. Another proposed theory of historical interest hypothesizes that naturally occurring DDAbs are present on the surface of certain platelet membrane glycoproteins but with weak affinity for self-antigens without causing clinical-pathological disease $[11,12]$. However, the presence of specific drugs can induce a much stronger reaction between the antigen and antibody. This may explain DITP that is noted even at low concentrations of oxaliplatin with strong antibody-platelet binding. This phenomenon may suggest that rather than a drug-induced conformational change on platelet membrane proteins resulting in neoepitopes, there is instead a high affinity binding between the drug and the complementarity-determining region (CDR) on the variable region of an antibody, which reconfigures the CDR to increase the specificity for a platelet glycoprotein binding site [14]. Our patient's serum also contained drug-independent antibodies, but we did not believe her to have idiopathic immune thrombocytopenia (ITP) since her presentation after multiple exposures (more than 10 cycles) was consistent with previously cited cases of oxaliplatin DITP.

It is of interest to further explore the mechanism behind each agent and whether the reaction represents two different mechanisms of DITP, multiple DDAbs, or if there is an antibody cross-reactivity between oxaliplatin and irinotecan which has not yet been investigated. Curtis et al. reported that complete absorption of one drug-specific antibody did not affect other DDAbs, suggesting the presence of multiple distinct DDAbs rather than a single antibody cross-reacting to different drugs. Patients treated with oxaliplatin are unusually prone to producing multiple DDAbs specific for drugs to which they are exposed. Usually, the clinical criteria are insufficient to make a diagnosis and confirmatory laboratory testing is needed. Therefore, there is a possibility that antibodies specific for additional drugs may have been present but were not checked [15]. Transfusion and pregnancy are major causes of alloimmunization, nondrug-dependent antibodies, and platelet refractoriness [16]. Pregnancy and previous transfusion can induce an HLA antibody; our patient did have a history of prior transfusion and previous pregnancies.

Better understanding of DITP will allow clinicians to readily distinguish it from other etiologies of thrombocytope- nia and minimize exposure of an offending drug. Other immune-mediated diseases with oxaliplatin have been reported such as Evan's syndrome [16] and oxaliplatininduced immune syndrome [13]. The development of multiple antibodies and antibody targets with the use of oxaliplatin carries clinical significance as it may limit the use of an otherwise versatile and effective therapeutic agent in colorectal cancer.

\section{Disclosure}

This case was previously reported as an abstract (February 25, 2016) and poster at the Eastern Regional Meeting Abstracts Display Posters (April 13, 2016).

\section{Conflicts of Interest}

There are no relevant conflicts of interest to be declared.

\section{References}

[1] T. Kam and M. Alexander, "Drug-induced immune thrombocytopenia," Journal of Pharmacy Practice, vol. 27, no. 5, pp. 430-439, 2014.

[2] B. R. Curtis, "Drug-induced immune thrombocytopenia: incidence, clinical features, laboratory testing, and pathogenic mechanisms," Immunohematology, vol. 30, no. 2, pp. 55-65, 2014.

[3] D. M. ARNOLD, S. KUKASWADIA, I. NAZI et al., "A systematic evaluation of laboratory testing for drug-induced immune thrombocytopenia," Journal of Thrombosis and Haemostasis, vol. 11, no. 1, pp. 169-176, 2013.

[4] A. Mitta, B. R. Curtis, J. A. Reese, and J. N. George, "Druginduced thrombocytopenia: 2019 update of clinical and laboratory data," American Journal of Hematology, vol. 94, pp. E76-E78, 2019.

[5] T. André, A. de Gramont, D. Vernerey et al., "Adjuvant fluorouracil, leucovorin, and oxaliplatin in stage II to III colon cancer: updated 10-year survival and outcomes according toBRAFMutation and Mismatch Repair Status of the MOSAIC Study," Journal of Clinical Oncology, vol. 33, no. 35, pp. 41764187, 2015.

[6] M. A. Bautista, W. T. Stevens, C. S. Chen, B. R. Curtis, R. H. Aster, and C. T. Hsueh, "Hypersensitivity reaction and acute immune-mediated thrombocytopenia from oxaliplatin: two case reports and a review of the literature," Journal of Hematology \& Oncology, vol. 3, no. 1, p. 12, 2010

[7] L. Bozec, P. Bierling, P. Fromont et al., "Irinotecan-induced immune thrombocytopenia," Annals of Oncology, vol. 9, no. 4, pp. 453-455, 1998.

[8] B. R. Curtis, J. Kaliszewski, M. B. Marques et al., "Immunemediated thrombocytopenia resulting from sensitivity to oxaliplatin," American Journal of Hematology, vol. 81, no. 3, pp. 193-198, 2006.

[9] R. Fontão-Wendel, P. M. Hoff, A. Lazar et al., "Immune-mediated pancytopenia induced by oxaliplatin: a case report," Transfusion, vol. 50, no. 7, pp. 1453-1459, 2010.

[10] B. R. Curtis, Y.-M. S. Hsu, N. Podoltsev et al., "Patients treated with oxaliplatin are at risk for thrombocytopenia caused by multiple drug-dependent antibodies," Blood, vol. 131, no. 13, pp. 1486-1489, 2018. 
[11] B. C. Mirtsching, J. N. George, R. H. Aster, and B. R. Curtis, "Irinotecan-induced immune thrombocytopenia," The American Journal of the Medical Sciences, vol. 347, no. 2, pp. 167169, 2014.

[12] K. Mittal, M. J. McNamara, B. R. Curtis, and K. R. McCrae, "Antiplatelet antibodies in oxaliplatin-induced immune thrombocytopenia," JRSM Open, vol. 5, no. 6, article 205427041453112, 2014.

[13] K. Bencardino, G. Mauri, A. Amatu et al., "Oxaliplatin immune-induced syndrome occurs with cumulative administration and rechallenge: Single Institution Series and Systematic Review Study," Clinical Colorectal Cancer, vol. 15, no. 3, pp. 213-221, 2016.

[14] E. James, N. Podoltsev, E. Salehi, B. R. Curtis, and M. W. Saif, "Oxaliplatin-induced immune thrombocytopenia: another cumulative dose-dependent side effect?," Clinical Colorectal Cancer, vol. 8, no. 4, pp. 220-224, 2009.

[15] S. E. Suh, M. J. Jang, S. Y. Chong, R. H. Aster, B. R. Curtis, and D. Oh, "A case of oxaliplatin-induced immune-mediated thrombocytopenia," Blood Research, vol. 49, no. 1, pp. 61-64, 2014.

[16] T. J. Legler, I. Fischer, J. Dittmann et al., "Frequency and causes of refractoriness in multiply transfused patients," Annals of Hematology, vol. 74, no. 4, pp. 185-189, 1997.

[17] A. Akdeniz, N. Kucukoztas, S. Yalcin, Z. Akcali, and O. Altundag, "Oxaliplatin-induced immune thrombocytopenia in a patient with colon cancer," The American Surgeon, vol. 77, no. 1, article E9, 2011.

[18] S. Buti, M. Riccò, M. D. Chiesa et al., "Oxaliplatin-induced hemolytic anemia during adjuvant treatment of a patient with colon cancer: a case report," Anti-Cancer Drugs, vol. 18, no. 3, pp. 297-300, 2007.

[19] F. Cobo, G. De Celis, A. Pereira, X. Latorre, J. Pujadas, and S. Albiol, "Oxaliplatin-induced immune hemolytic anemia: a case report and review of the literature," Anti-Cancer Drugs, vol. 18, no. 8, pp. 973-976, 2007.

[20] C. C. Earle, W. Y. Chen, D. P. Ryan, and R. J. Mayer, "Oxaliplatin-induced Evan's syndrome," British Journal of Cancer, vol. 84, no. 3, p. 441, 2001.

[21] A. K. Koutras, T. Makatsoris, F. Paliogianni et al., "Oxaliplatininduced acute-onset thrombocytopenia, hemorrhage and hemolysis," Oncology, vol. 67, no. 2, pp. 179-182, 2004.

[22] S. Ohta, Y. Cho, S. Oshima, O. Hosoya, K. Juni, and H. Kojima, "Oxaliplatin-induced acute-onset thrombocytopenia and hemorrhage: case report and review of the literature," Oncology Letters, vol. 3, no. 6, pp. 1297-1300, 2012.

[23] M. Pavic, P. Moncharmont, P. Sève, D. Rigal, and C. Broussolle, "Oxaliplatin-induced immune thrombocytopenia," Gastroentérologie Clinique et Biologique, vol. 30, no. 5, pp. 797-798, 2006.

[24] M. W. Saif, A. The, and L. Ledbetter, "Oxaliplatin-mediated autoimmune thrombocytopenia," Clinical Colorectal Cancer, vol. 8, no. 1, pp. 61-62, 2009.

[25] M. Santodirocco, V. Lombardi, C. Fesce, G. Palumbo, S. Capalbo, and M. Landriscina, "Life-threatening oxaliplatininduced acute thrombocytopenia, hemolysis and bleeding: a case report," Acta Oncologica, vol. 47, no. 8, pp. 1602-1604, 2008.
[26] H. Schade, L. Davis, and P. Kabos, "Middle-aged man with acute thrombocytopenia subsequent to fluorouracil and oxaliplatin chemotherapy for colorectal cancer," Oncology, vol. 30, no. 11, pp. 992-994, 2016, 1000-1.

[27] Y. Y. Shao and R. L. Hong, "Fatal thrombocytopenia after oxaliplatin-based chemotherapy," Anticancer Research, vol. 28, no. 5B, pp. 3115-3117, 2008.

[28] K. Suzuki, H. Oda, Y. Sugawara et al., "Oxaliplatin-induced acute thrombocytopenia: a case report and review of the literature," Internal Medicine, vol. 52, no. 5, pp. 611-615, 2013.

[29] B. M. Taleghani, S. Fontana, O. Meyer et al., "Oxaliplatininduced immune pancytopenia," Transfusion, vol. 45, no. 5, pp. 704-708, 2005.

[30] S. A. Curtis, B. R. Curtis, A. I. Lee, J. E. Hendrickson, J. Lacy, and N. A. Podoltsev, "A patient with oxaliplatin immuneinduced syndrome (OIIS) who also developed leucovorin and palonosetron-associated thrombocytopenia," Hematology, vol. 23, no. 7, pp. 429-432, 2018.

[31] E. Pan, E. Hsieh, and C. Piatek, "Case report: oxaliplatininduced immune-mediated thrombocytopenia," Case Reports in Oncology, vol. 11, no. 3, pp. 880-882, 2018. 


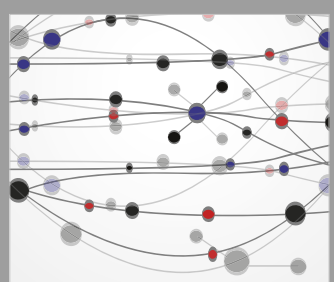

The Scientific World Journal
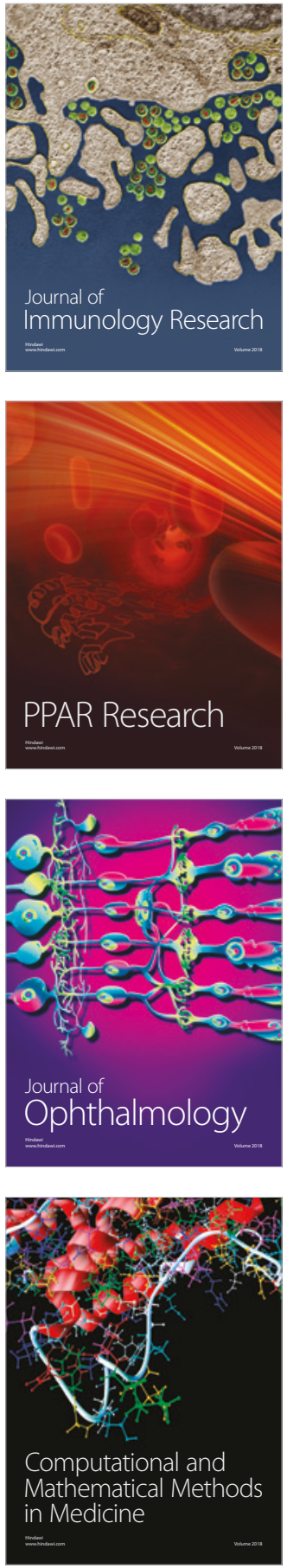

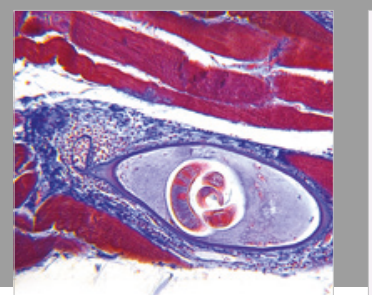

Gastroenterology Research and Practice

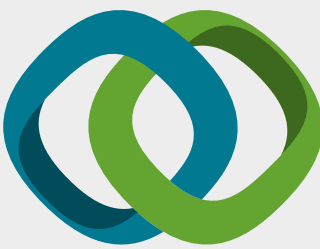

\section{Hindawi}

Submit your manuscripts at

www.hindawi.com
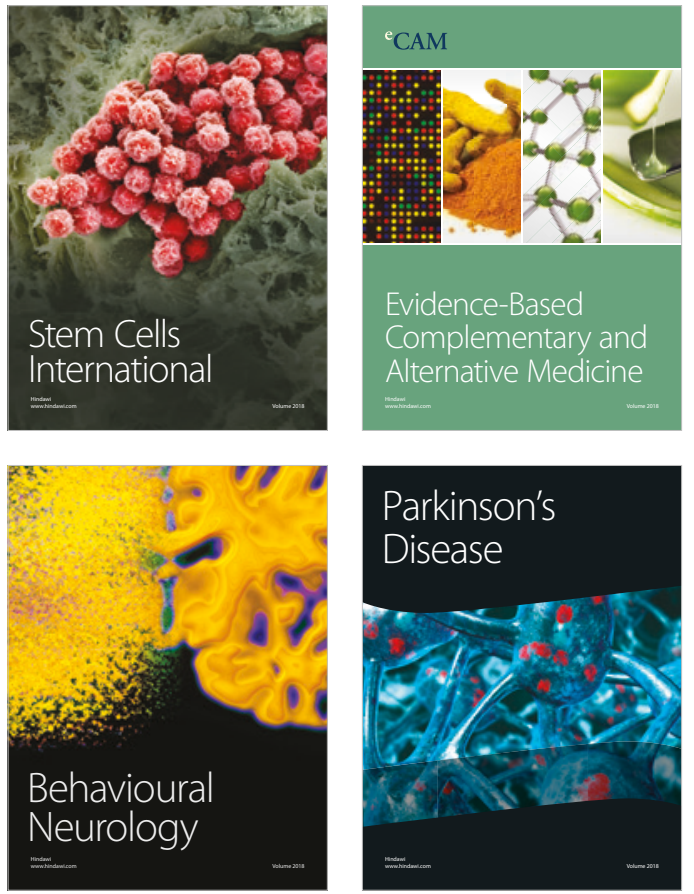

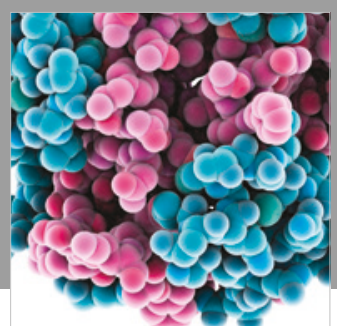

ournal of

Diabetes Research

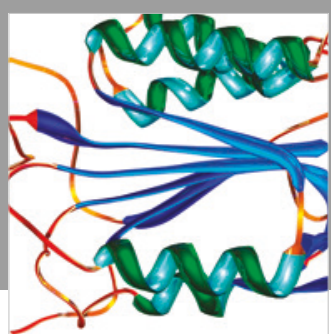

Disease Markers
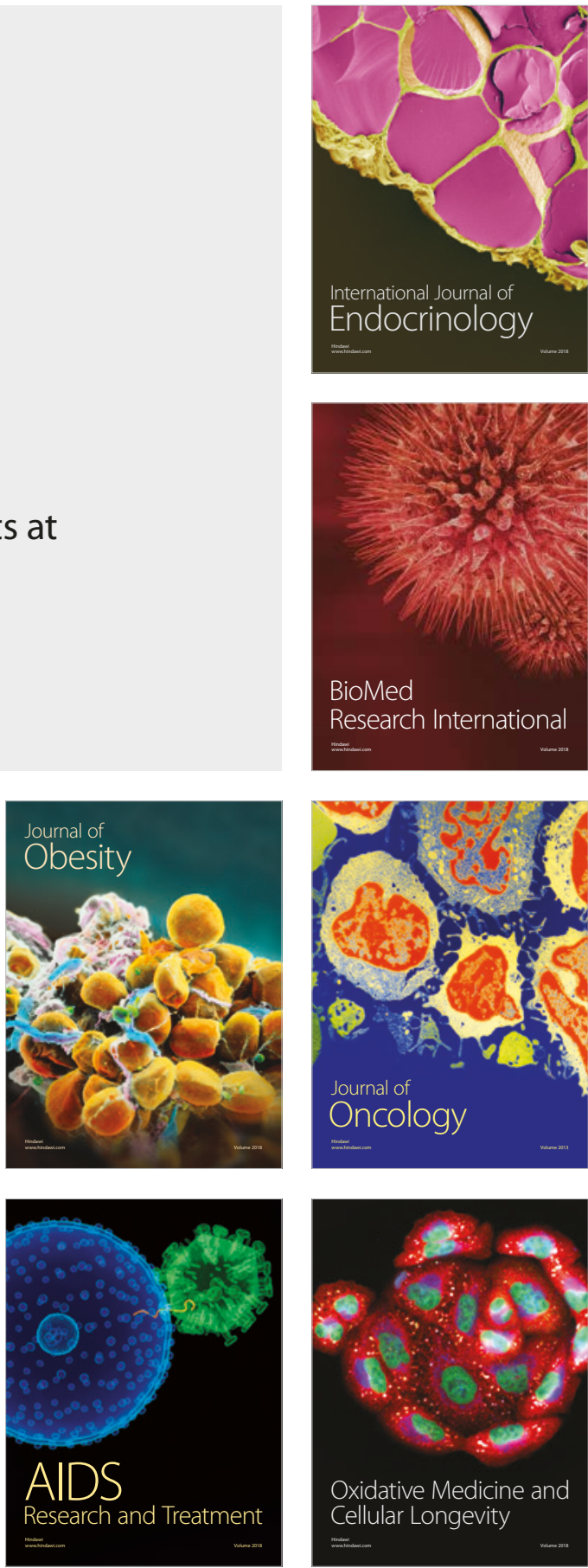